\title{
Scaling in Small-World Resistor Networks
}

\author{
G. Korniss ${ }^{\text {a,* }}$, M.B. Hastings ${ }^{b}$, K.E. Bassler ${ }^{c}$, \\ M.J. Berryman ${ }^{d}$, B. Kozma ${ }^{a}$, D. Abbott ${ }^{d}$ \\ ${ }^{a}$ Department of Physics, Applied Physics, and Astronomy, Rensselaer Polytechnic \\ Institute, $1108^{\text {th }}$ Street, Troy, NY 12180-3590, USA \\ ${ }^{\mathrm{b}}$ Center for Non-linear Studies and Theoretical Division, Los Alamos National \\ Laboratory, Los Alamos, NM 87545, USA \\ ${ }^{\mathrm{c}}$ Department of Physics, 617 Science and Research Blvd I, Univesity of Houston, \\ Houston, TX 77204-5005, USA \\ ${ }^{\mathrm{d}}$ Centre for Biomedical Engineering (CBME) and School of Electrical $\mathscr{G}$ Electronic \\ Engineering, The University of Adelaide, SA 5005, Australia
}

\begin{abstract}
We study the effective resistance of small-world resistor networks. Utilizing recent analytic results for the propagator of the Edwards-Wilkinson process on small-world networks, we obtain the asymptotic behavior of the disorder-averaged two-point resistance in the large system-size limit. We find that the small-world structure suppresses large network resistances: both the average resistance and its standard deviation approaches a finite value in the large system-size limit for any non-zero density of random links. We also consider a scenario where the link conductance decays as a power of the length of the random links, $l^{-\alpha}$. In this case we find that the average effective system resistance diverges for any non-zero value of $\alpha$.
\end{abstract}

Key words: Small-world model, Resistor networks, Scaling PACS: 89.75.Hc, 05.60.Cd

Resistor networks have been widely studied since the 70's as models for conductivity problems and classical transport in disordered media $[1,2,3,4]$. Related studies on fuse networks have been investigated on random percolating lattices with various applications to breakdown processes in condensed matter and materials systems, ranging from brittle fracture to dielectric breakdown $[5,6,7,8,9]$.

\footnotetext{
* E-mail: korniss@rpi.edu
} 
Recent research on complex networks $[10,11,12]$ has turned to focus on dynamics on networks with applications to synchronization in natural and artificial systems $[13,14,15,16,17,18]$, and transport phenomena $[19,20,21,22]$. Interesting recent studies have examined the tradeoffs between redundancy and pleiotropy [23], and centralized versus decentralized design [24], in complex networks. Finding the resistance between any two points on a complex network is tractable and builds upon early mesh-resistance techniques [25]. Estimating the strength of collaborative ties between nodes in collaboration networks [26] and quantifying the centrality of a node in weighted networks can also be modeled by resistor networks [27]. While resistor networks have been employed to study and explore community structures in social networks [27,28,29], they have not been investigated as prototypical models for transport phenomena in complex networks until very recently [30,31]. The work by López et al. [30] revealed that in scale-free (SF) networks [10,32] anomalous transport properties can emerge, displayed by the power-law tail of distribution of the network conductance.

Here we investigate the effective system resistance of small-world (SW) networks $[33,34,35]$. Our results, in part, are based on recent calculations [36,37,38,39] of the disordered averaged propagator of the Edwards-Wilkinson (EW) [40] process extended to a SW network. The EW process on a network can be thought of in terms of a synchronization paradigm in a noisy environment. As a linear approximation, it also serves as the simplest model for generic causallyconstrained queuing networks [41], such as manufacturing supply chains, ecommerce based services facilitated by interconnected servers [42], and certain distributed parallel schemes on computer networks $[18,43]$. In the context of the latter, it was shown [18] that when extending the original short-range connections to a SW-like network (essentially, by adding a small density of random links on top of a regular graph), the spread between completion times of tasks performed on different nodes of a computer network remains bounded, rather than diverging over time. Further, an infinitesimal extra "cost" is sufficient to achieve this reduction. An important measure of efficiency is the spread (or "width") of task-completion landscapes in such processing networks (larger spread corresponds to longer delays and poorer efficiency). It is evident that this measure - the width of the EW landscape on a network - is identical to the average resistance (characterizing transport efficiency) of the same network. While this connection between the network propagator and the network resistance $[2,44,45]$, just like the one between random walks and network resistance $[46,47,48]$, is well known, it has not been exploited to study transport efficiency of SW networks. Further, the connection between the average spread of an EW steady-state landscape and the resistance of the same network gives some insight in treating synchronization and transport efficiency on the same footing. Namely, understanding the effects of the SW links in suppressing the diverging long-wavelength modes of the network propagator, originally present in regular lattices. 
Our main result is that in SW networks, the average system resistance becomes finite for an arbitrarily small density of random links, governed by the same behavior of the network propagator which is responsible for suppressing "rough" synchronization landscapes $[18,36]$.

The Edwards-Wilkinson process on a network. - The EW process in a synchronization context on a network, is given by the Langevin equation

$$
\partial_{t} h_{i}=-\sum_{j=1}^{N} A_{i j}\left(h_{i}-h_{j}\right)+\eta_{i}(t)
$$

where $h_{i}(t)$ is the general stochastic field variable on a node (such as fluctuations in the task-completion landscape in certain distributed parallel schemes on computer networks $[18,36])$ and $\eta_{i}(t)$ is a delta-correlated noise with zero mean and variance $\left\langle\eta_{i}(t) \eta_{j}\left(t^{\prime}\right)\right\rangle=2 \delta_{i j} \delta\left(t-t^{\prime}\right)$. Here, $A_{i j}=A_{j i}>0$ is the effective coupling between the nodes $\left(A_{i i} \equiv 0\right)$. Defining the network Laplacian, $\Gamma_{i j}=\delta_{i j} \sum_{l} A_{i l}-A_{i j}$, we can rewrite Eq. (1)

$$
\partial_{t} h_{i}=-\sum_{j=1}^{N} \Gamma_{i j} h_{j}+\eta_{i}(t)
$$

For the steady-state equal-time two-point correlation function one finds

$$
G_{i j} \equiv\left\langle\left(h_{i}-\bar{h}\right)\left(h_{j}-\bar{h}\right)\right\rangle=\hat{\Gamma}_{i j}^{-1}=\sum_{k=1}^{N-1} \frac{1}{\lambda_{k}} \psi_{k i} \psi_{k j},
$$

where $\bar{h}=(1 / N) \sum_{i=1}^{N} h_{i}$ and $\langle\ldots\rangle$ denotes an ensemble average over the noise in Eq. (2). Here, $\hat{\Gamma}^{-1}$ denotes the inverse of $\Gamma$ in the space orthogonal to the zero mode. Also, $\left\{\psi_{k i}\right\}_{i=1}^{N}$ and $\lambda_{k}, k=0,1, \ldots, N-1$, denote the $k$ th normalized eigenvectors and the corresponding eigenvalues, respectively. The $k=0$ index is reserved for the zero mode of the Laplacian on any network: all components are identical of this eigenvector and $\lambda_{0}=0$. The last form in Eq. (3) (the spectral decomposition of $\hat{\Gamma}^{-1}$ ) is useful for exact numerical diagonalization purposes. As one can see from Eq. (3), $G$ is the inverse of the coupling matrix $\Gamma$ in the space orthogonal to the zero mode of the Laplacian. In particular, the average spread or width in the synchronization landscape becomes

$$
\left\langle w^{2}\right\rangle=\left\langle\frac{1}{N} \sum_{i=1}^{N}\left(h_{i}-\bar{h}\right)^{2}\right\rangle=\frac{1}{N} \sum_{i=1}^{N} G_{i i}=\frac{1}{N} \sum_{k=1}^{N-1} \frac{1}{\lambda_{k}} .
$$

For large networked systems, the above observable is typically self-averaging $\left\langle w^{2}\right\rangle \simeq\left[\left\langle w^{2}\right\rangle\right]$, where $[. .$.$] denotes the average over the network disorder. Thus,$ 
if one is able to calculate the disorder-averaged propagator $\left[G_{i j}\right]$, it provides the scaling behavior of the average spread of the synchronization landscape in the limit of $N \rightarrow \infty$.

The two-point resistance of a network. - The stationary currents and voltages in any network of resistors are governed by Kirchhoff's and Ohm's laws

$$
\sum_{n} A_{m n}\left(V_{m}-V_{n}\right)=I_{m}
$$

where $A_{m n}$ is the conductance of the link between node $m$ and $n$, and $I_{m}$ is the net current flowing into the network at node $m$. Note that $I_{m}$ is zero, unless node $m$ is connected to an external terminal. Connecting the network to a "battery" with fixed voltage drop $V$ through nodes $i$ and $j$ as the input and output terminals, yields

$$
\sum_{n} \Gamma_{m n} V_{n}=I\left(\delta_{m i}-\delta_{m j}\right)
$$

where $\Gamma_{m n}$ is the same network Laplacian as introduced earlier in the context of the EW process [Eq. (2)], associating the link conductance with the coupling matrix there; $\delta_{i j}$ is the Kronecker delta. Here, $I$ is the magnitude of the current entering and leaving the system at node $i$ and node $j$, respectively. Solving for the voltages is well defined in the subspace orthogonal to the zero-mode of the network Laplacian; the right-hand side vector of Eq. (6) is in that subspace. Hence, introducing the voltages measured from the mean $\hat{V}_{m}=V_{m}-\bar{V}$, where $\bar{V}=(1 / N) \sum_{m=1}^{N} V_{m}$, and employing $\hat{\Gamma}^{-1}$ one has

$$
\hat{V}_{m}=\sum_{n} \hat{\Gamma}_{m n}^{-1} I_{n}=\sum_{n} \hat{\Gamma}_{m n}^{-1} I\left(\delta_{n i}-\delta_{n j}\right)=I\left(G_{m i}-G_{m j}\right)
$$

where $G$ is the same network propagator discussed in the previous section in the context of the EW process on networks [Eq. (3)]. Applying the above equation to the voltage difference across node $i$ and node $j$ to which the battery is attached, one finds

$$
V=V_{i}-V_{j}=\hat{V}_{i}-\hat{V}_{j}=I\left(G_{i i}+G_{j j}-2 G_{i j}\right)
$$

For the equivalent two-point resistance between node $i$ and $j$ one finally obtains

$$
R_{i j} \equiv \frac{V}{I}=G_{i i}+G_{j j}-2 G_{i j}=\sum_{k=1}^{N-1} \frac{1}{\lambda_{k}}\left(\psi_{k i}^{2}+\psi_{k j}^{2}-2 \psi_{k i} \psi_{k j}\right)
$$


where the last form in Eq. (9) is, again, useful for exact numerical diagonalization purposes. Looking at Eq. (9), one can realize that the two-point resistance of a network between node $i$ and $j$ is the same as the steady-state height-difference correlation function of the EW process on the network

$$
\left\langle\left(h_{i}-h_{j}\right)^{2}\right\rangle=G_{i i}+G_{j j}-2 G_{i j}=R_{i j} .
$$

The height-difference correlation function is a standard observable in surfacegrowth phenomena, extensively studied in the past two decades [49], so many of the answers for regular resistor networks can be obtained directly by looking at the equivalent EW model on a $d$-dimensional substrate. For example, in an infinite one-dimensional system, the resistance between two nodes, separated by a distance $|i-j|$, diverges with the separation as $R_{i j}=R(|i-j|) \simeq|i-j|$ $[44,45,49]$. Another trivial, but insightful, relationship between the EW process and the resistor network can be obtained by summing up Eq. (10) over all $i \neq j$ pairs, yielding

$$
\bar{R} \equiv \frac{1}{N(N-1)} \sum_{i \neq j} R_{i j}=2\left\langle w^{2}\right\rangle,
$$

i.e., the average system resistance of a given network is twice the steady-state width of the EW process on the same network.

Effective Resistance of simple SW Networks. -First, we consider "simple" SW resistor networks, where the conductance of each link is identical, with unit value, for simplicity. When studying network-transport phenomena for systems where physical links are subject to strong cost and geometric constraints, this can be unrealistic and cost-prohibitive. For others, e.g., modeling information flow in social networks $[27,28,29,50]$, this can be an acceptable starting point, since "long-range" connections do not necessarily degrade the information carrying capacity and the efficiency (e.g., influence) of that link. We start with a one-dimensional ring with $N$ nodes (i.e., impose periodic boundary conditions), and add a "random" link to each pair of nodes, independently for each pair, with probability $p / N$. In addition to the two nearest-neighbor connections, now each node, on average has $p$ random links, so $p$ is the density of random links. The resulting network is essentially an Erdős-Rényi (ER) network [51] on top of a one-dimensional graph. This SW construction slightly differs from the original Watts-Strogatz one [33] where random links are introduced through "rewiring". The resulting network, however, has the same universal properties in the small- $p$, large- $N$ limit $[52,53]$, and is also more amenable to analytic approximations.

The coupling matrix for the differences of the relevant variables [Eq. (1) and 
(5)] then becomes

$$
A_{i j}=\delta_{i, j-1}+\delta_{i, j+1}+J_{i j}
$$

where the matrix elements $J_{i j}$ are quenched random variables; $J_{i j}=1$ with probability $p / N$ and $J_{i j}=0$ with probability $(1-p / N)$. The corresponding Laplacian then can be written as

$$
\Gamma_{i j}=2 \delta_{i, j}-\delta_{i, j-1}-\delta_{i, j+1}+\delta_{i j} \sum_{l} J_{i l}-J_{i j}
$$

Equations (12) and (13), with $J_{i j}$ defined above, correspond to identical (unit) conductance for each existing connection in the resistor network. Our numerical scheme relied on the exact numerical diagonalization of the SW network Laplacian $\Gamma$ in Eq. (13) [54]. Our analytic results, asymptotically exact in the large system-size limit, are straightforward applications of those of the EW propagator on SW networks [36,37,38,39].

Averaging over the network-disorder restores translational invariance, hence the disorder-averaged two-point function $\left[G_{i j}\right]=[G(|i-j|)]$ will only depend on the underlying Euclidean distance between the nodes. These correlation functions have been calculated using disorder-averaged self-consistent perturbation theory $[36,37,38,39]$. For the disorder-averaged two-point function for small $p$ values, in the infinite system-size limit one finds $[36,37,38,39]$

$$
[G(l)] \simeq \frac{1}{2 \sqrt{\Sigma}} e^{-\sqrt{\Sigma} l}
$$

where $\Sigma \sim p^{2}$ is an effective mass generated by the random links for simple SW networks [36,53]. Then, using Eq. (9), for the average resistance on a SW network between two nodes separated by a distance $l$, we obtain

$$
[R(l)]=2([G(0)]-[G(l)]) \simeq \frac{1}{\sqrt{\Sigma}}\left(1-e^{-\sqrt{\Sigma} l}\right)
$$

approaching a finite value in the limit of infinite separation, $\lim _{l \rightarrow \infty}[R(l)]=$ $\Sigma^{-1 / 2} \sim p^{-1}$. In contrast, on a regular one-dimensional ring, the resistance between two nodes separated by a distance $l$ diverges in a power-law fashion, $R(l) \simeq l$, as can be seen by taking the $\Sigma \rightarrow 0$ limit in Eq. (15) or by direct calculations on regular lattices $[44,45]$. Further, the average resistance is finite for an arbitrarily small but non-zero $p$ in the limit of $N \rightarrow \infty$,

$$
\bar{R} \simeq[\bar{R}]=2\left[\left\langle w^{2}\right\rangle\right]=2[G(0)] \simeq \frac{1}{\sqrt{\Sigma}} \sim p^{-1}
$$



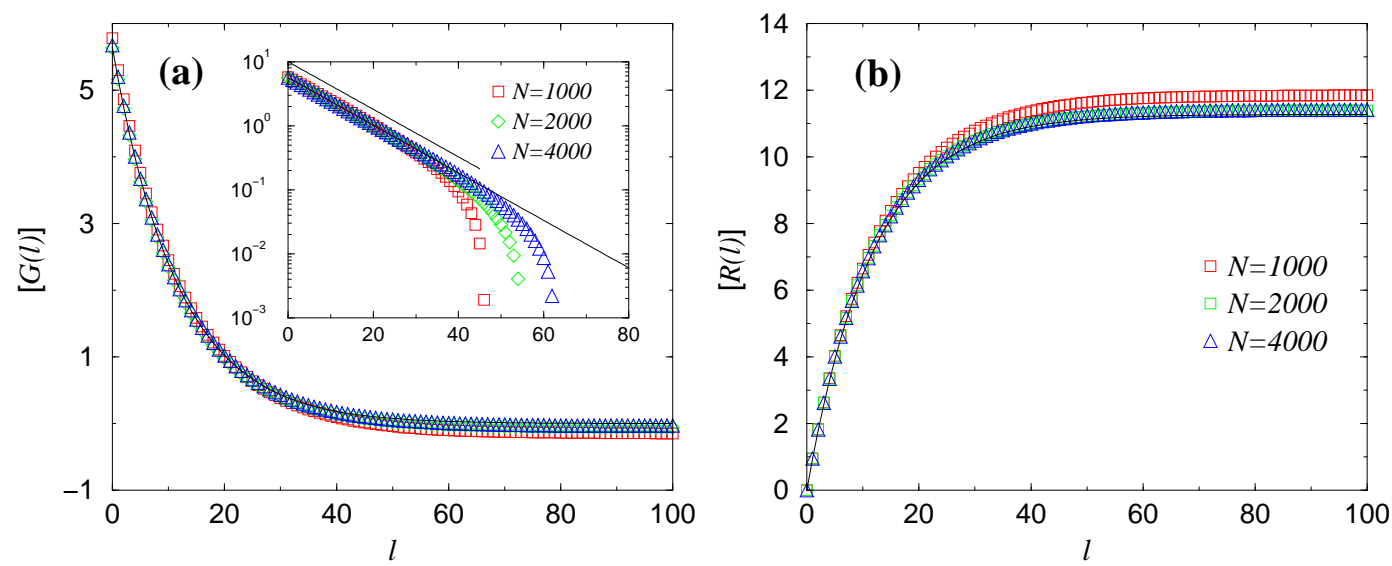

Fig. 1. Disorder-averaged (a) two-point function and (b) two-point resistance as a function of the separation $l$ in simple SW networks for $p=0.10$ and three system sizes. The solid line in (a) and (b) corresponds to the exponential decay and saturation given by Eqs. (14) and (15), respectively
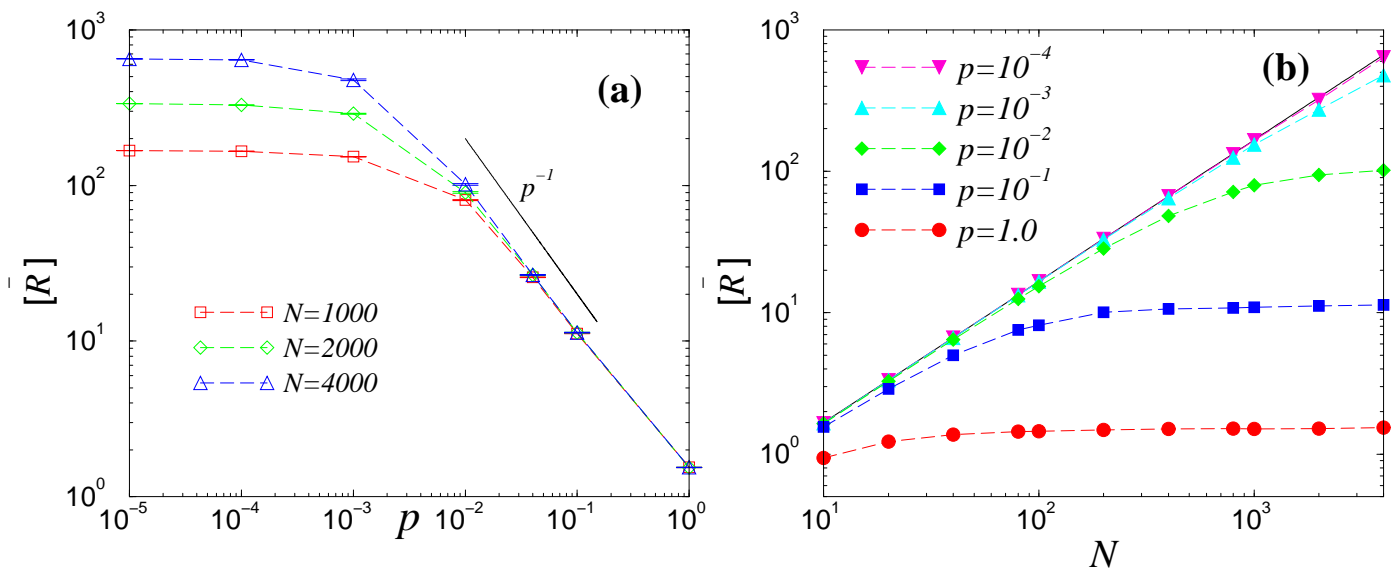

Fig. 2. (a) Average resistance vs the density of random links. The straight solid line indicate the asymptotic infinite system-size behavior [Eq. (16)]. (b) Average resistance vs the system size in simple SW networks. The straight solid line corresponds to the behavior of the one-dimensional regular network (ring), $[\hat{R}] \simeq N / 6$.

in strong contrast with average resistance for a regular network diverging as $\bar{R} \simeq N / 6$. Equations (15) and (16) are the central results of our paper. They capture the average resistance between nodes separated by a distance $l$ and the average system resistance for SW networks with a small but non-zero density of random links, respectively. Results from exact numerical diagonalizations, shown in Fig. 1 and Fig. 2, up to systematic finite-size effects, agree very well with the above predictions.

In addition to the above asymptotic results, valid in the infinite system-size limit, we also constructed the scaling form, capturing the finite-size effects, e.g., for the average resistance $[38,55]$. From the above it is clear that in addition to the linear system size $N$, there is one other lengthscale in the problem for non-zero $p$ values, $\xi=1 / \sqrt{\Sigma} \sim p^{-1}$. This lengthscale is, in fact, 


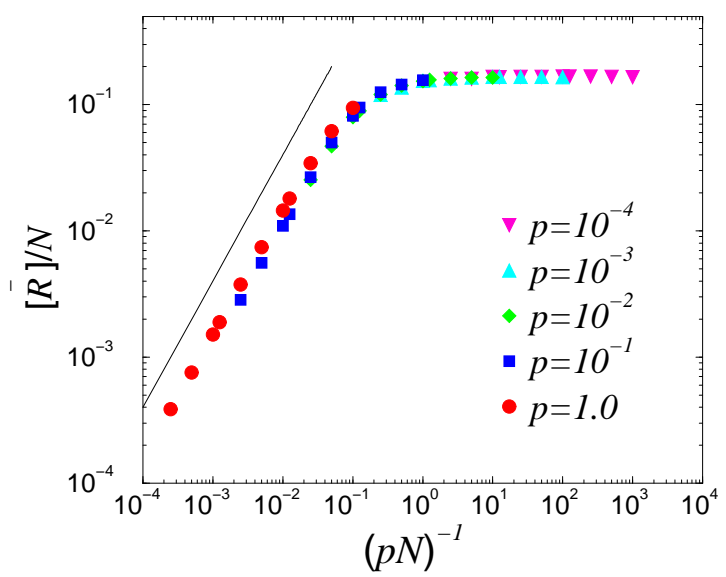

Fig. 3. Scaled average resistance in simple SW networks according to Eq. (17). The straight solid line corresponds to the asymptotic behavior of the scaling function for small arguments [Eq. (18)].

the average distance between nodes which have random links emanating from them. For $p=0$ (the limit of a regular one-dimensional network) $[\bar{R}] \sim N$, while for $p \neq 0$, in the infinite system-size limit, it approaches a constant, $[\bar{R}] \simeq 1 / \sqrt{\Sigma}=\xi \sim p^{-1}$ [Fig. 2(a) and (b)]. Thus, the finite-size behavior of the average resistance can be expressed as

$$
[\bar{R}]=N f(\xi / N)
$$

where $f(x)$ is a scaling function such that

$$
f(x) \sim \begin{cases}x & \text { if } x \ll 1 \\ \text { const. if } x \gg 1\end{cases}
$$

The scaled numerical data, $[\bar{R}] / N$ vs $\xi / N$ [Fig. 3], shows good collapse, as suggested by Eq. (17).

We also studied the probability distribution of the effective resistance of the network [Fig. 4]. The overall distribution is shown in Fig. 4(a). Further, we constructed the distribution of the effective network resistance between two nodes separated by a distance $l, P(R \mid l)[4]$, indicating that they converge to a limit distribution for $l \rightarrow \infty$ [Fig. 4(b) and (c)]. These results imply that for SW networks, both small and large effective resistance values are strongly (at least exponentially) suppressed about the average. This is in strong contrast with the behavior of SF resistor networks [30], where large resistance values are strongly suppressed, but the probability of small values decays only in a power-law fashion; hence a power-law tail in the conductance distribution occurs for large $g \equiv 1 / R$ values. This finding for SF networks implies [30], that there exist a few nodes ("hubs") in the system that, if selected as the input and 

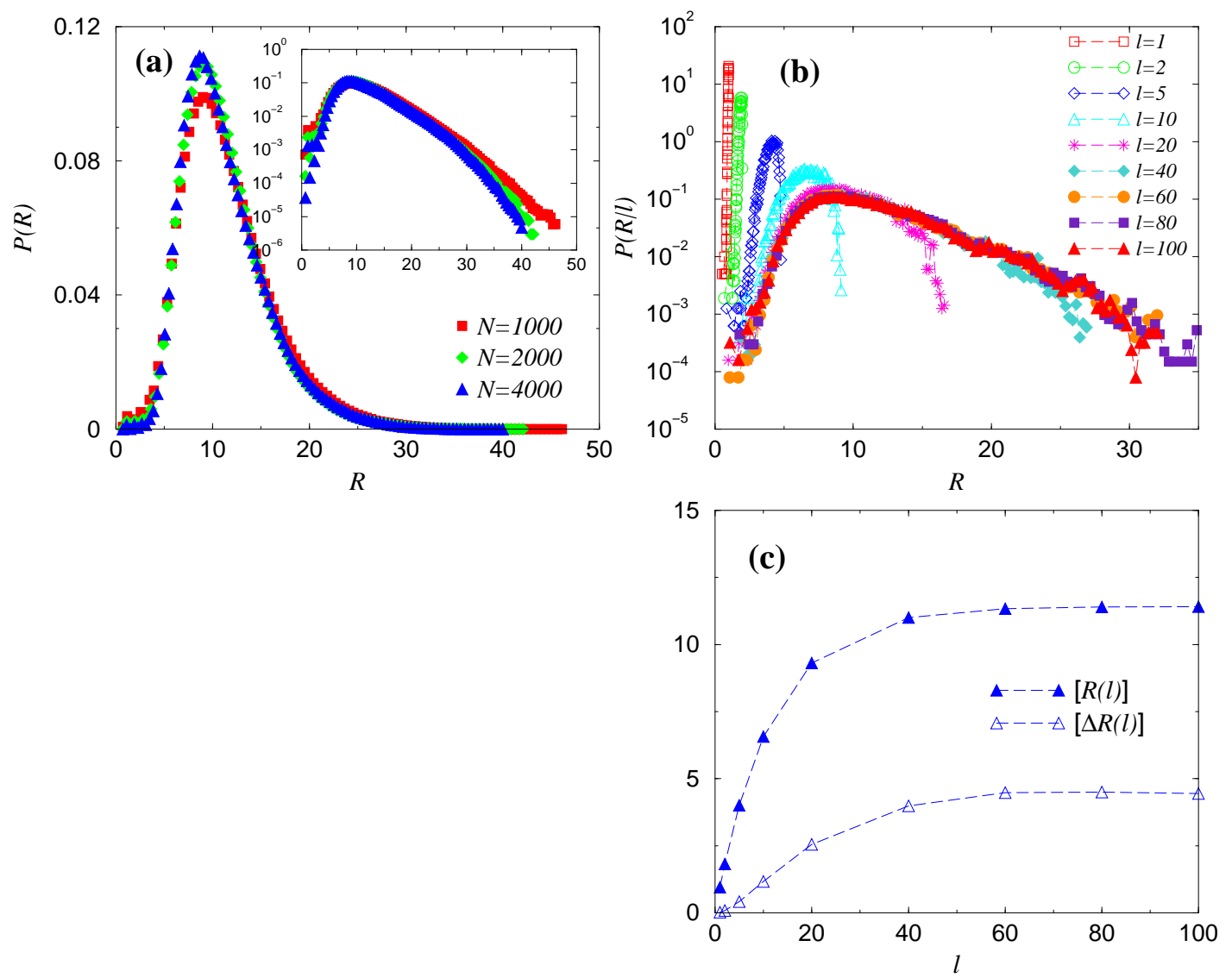

Fig. 4. (a) Network resistance probability distributions (histograms) for $p=0.10$ and for three system sizes. (b) Network resistance probability distributions (histograms) for nodes separated by a distance $l$, for various $l$ values for $N=4000$. (c) Behavior of the average $[R(l)]$ and the standard deviation $[\Delta R(l)]$ of the distributions shown in (b).

output nodes, can support anomalously large transport through the network. This phenomenon is absent (as one can expect) in SW networks, just like in completely random (ER) networks [30] related to the exponential tail of the degree distributions of these networks. In Fig. 5 we compare the conductance distributions for the SW and the Barabási-Albert (BA) [32,56] SF network, with the same average degree and uniform link conductance. At this point we note that while anomalously large conductances are absent in SW networks, they are more efficient, on average, in supporting transport between two arbitrary pairs of nodes, i.e., $[R]_{S W}<[R]_{S F}$, and $[g]_{S W}>[g]_{S F}$. In comparison, for the two networks shown in Fig. 5 with the same average degree and uniform link conductance, the average network resistance and conductance values are $[R]_{S W} \simeq 0.472,[R]_{S F} \simeq 0.572$, and $[g]_{S W} \simeq 2.28,[g]_{S F} \simeq 1.93$, respectively. In real-life complex networks with SF structure, however, the link conductances are typically weighted [21], ultimately leading to better performance for SF networks [30].

Effective Resistance of $S W$ Networks with Distance-Dependent Conductances.- 


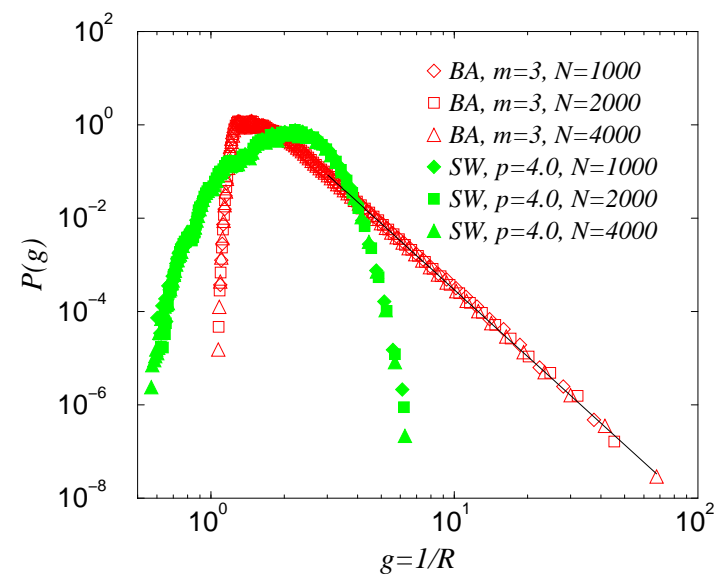

Fig. 5. Comparison of the network conductance distribution with that of the BA scale-free network model with the same average degree $\langle k\rangle=6$ (BA network with $m=3[56]$, and SW network with $p=4.0$.)

Now we consider the more general case where the conductances of the random (possibly long-range) links decay with the underlying spatial distance between the nodes they connect in a power-law fashion: $J_{i j}=1 /|i-j|^{\alpha}$ with probability $p / N$ and $J_{i j}=0$ with probability $(1-p / N)$ in Eq. (12). Keeping the density of the random links, $p$, fixed (at a non-zero value) and taking the large system-size limit, corresponds to the limit of fixed density of weak links at large scales. Then one can argue that, to leading order, mean-field scaling holds $[37,38,39,55,57]$. Focusing on the $0 \leq \alpha \leq 1$ regime, we find that the average link strength, decaying as $\left[J_{i j}\right]=(p / N)|i-j|^{-\alpha}$, gives rise to a system-size dependent effective mass $\Sigma_{N} \simeq(N / 2)^{-\alpha} p /(1-\alpha)$ and consequently

$$
[\bar{R}] \simeq \Sigma_{N}^{-1 / 2} \sim N^{\alpha / 2}
$$

Thus, the average system resistance diverges with the system size for an arbitrarily small but nonzero value of $\alpha$. Figure 6 supports this picture, but also indicates that corrections beyond the mean-field approximation are important and noticeable for the range of system-sizes that were accessible via numerical methods. In fact, an analysis of the naive perturbative approach [37] reveals that although higher-order corrections are becoming progressively smaller as $N$ increases, their prefactor is singular for certain values of $\alpha$ [55]. Given these subtleties, the deviations [Figure 6] from the predicted asymptotic scaling Eq. (19) are reasonable.

For the interested reader, familiar with the diagrammatics of [36], we present a brief analysis of the higher order corrections. The mean-field gives a selfenergy of order $N^{-\alpha}$. There are some corrections for finite $N$ to Eq. (15), but the most important higher-order ones (higher order in powers of $N^{-1}$ compared to the mean-field) are corrections to the self-energy. The leading order correction to the mean-field involves diagrams in which a single link 


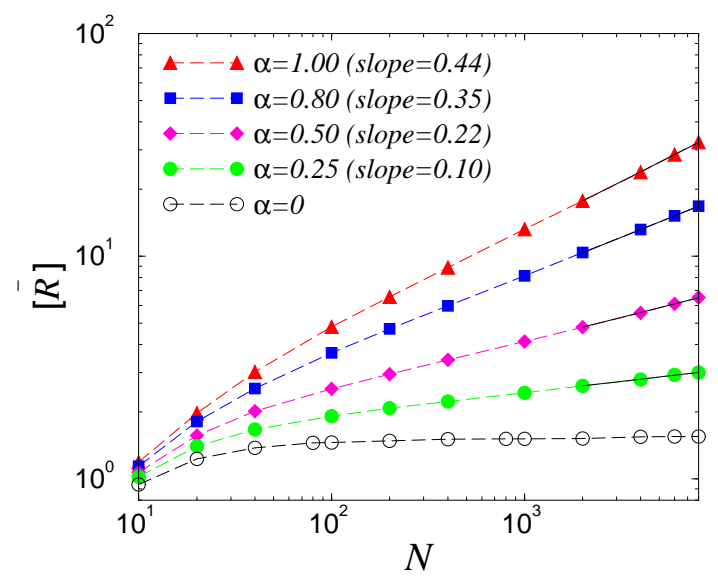

Fig. 6. Average system resistance of SW networks with distance-dependent conductances as described in the text, for $p=1.0$ for different $\alpha$ values. The solid line segments are the measured slopes for $\alpha \neq 0$, with their values shown in the legends; these values should be compared to $\alpha / 2$, the exponent of the leading-order perturbative results [Eq. (19)].

appears twice; these diagrams involve summing over the length of the link and are mulitplied by the strength of the link squared: $|i-j|^{-2 \alpha}$. For $\alpha<1 / 2$, this sum diverges for large $N$ and gives rise to corrections to the mean-field $\Sigma$, so that $\Sigma_{0}=p(N / 2)^{-\alpha} /(1-\alpha)$ is the mean-field value and the leading correction is $\Sigma=p(N / 2)^{-\alpha} /(1-\alpha)-p\left[(N / 2)^{-2 \alpha} /(1-2 \alpha)\right] / \sqrt{\Sigma_{0}}+\ldots$. Using this correction to $\Sigma$, the corrections to the resistance are of order $N^{0}$, and thus for $\alpha<1 / 2$ may be significant compared to the value in Eq. (19). The coefficient of these corrections becomes singular at $\alpha=1 / 2$ and for $\alpha \geq 1 / 2$, the self-energy $\Sigma$ becomes non-local and acquires a momentum dependence, which may be shown to change the form of the higher-order corrections.

Also note that this behavior is very different from that of the case where the strength (conductance) of the random links is uniform, but the probability of connecting two nodes, separated by a distance $l$, decays as $l^{-\alpha}[37,38,58]$. There exists a finite region, $0<\alpha<2$, where the propagator, hence in the context of this paper, the average system resistance, remains finite in the limit of $N \rightarrow \infty$. In the present case, where the link-length distribution is uniform, but the link strength decays as $l^{-\alpha}$, the average system resistance is finite only for $\alpha=0$.

This contrasting behavior between the two different " $l$ " " implementations of the random links (strength vs probability) is in accord with recent studies on phase transition on SW networks. Interacting systems often exhibit mean-fieldlike phase transitions $[57,59,60,61,62,63,64,65]$, even for an arbitrarily small but nonzero density of random links added to a one-dimensional regular graph. However, in the case of the strength of the random links decaying in the above $l^{-\alpha}$ fashion, for the Ising model on SW networks, it was shown [66] that no phase transition occurs at any finite temperature for any nonzero $\alpha$. 
Summary and Outlook. - We obtained the scaling behavior of the effective resistance of SW networks. For uniform link conductances, we found that for an arbitrary small density of random links, the average system resistance is finite, and the two-point resistance, as function of the distance between the nodes, saturates exponentially fast to the same finite value. When the link conductance decays with the distance as $l^{-\alpha}$, the average network resistance diverges with the number of nodes as $N^{\alpha / 2}$.

Ultimately, one is interested not only in the global transport or flow characteristics of the network, but also in their effect on the local "components", capacity limitations, and possible global network failures. In the context of resistor networks, the question of voltage landscapes in the network, or more specifically, the voltage-drop distribution across the links, can be addressed. Such a study can reveal the most vulnerable links/connections to be "blown" when increasing the overall load in the network. In particular, studying the properties of the extreme (largest) voltage-drops across the links in the network carries information on the weakest links of the network, and in turn, provides solutions from a system-design viewpoint. Fuse networks have been intensively studied on random percolating lattices with various applications to breakdown processes in condensed matter and materials systems, ranging from brittle fracture to dielectric breakdown $[5,6,7,8,9]$. Future work will address these questions from a general complex network vulnerability viewpoint $[67,68,69]$.

Acknowledgments. - GK and BK were supported by NSF Grant No. DMR0426488 and the Research Corporation, MJB and DA by the Australian Research Council (ARC), MBH by US DOE W-7405-ENG-36, and KEB by NSF Grant No. DMR-0427538 and the Alfred P. Sloan Foundation.

\section{References}

[1] S. Kirkpatrick, Phys. Rev. Lett. 27, 1722 (1971).

[2] S. Kirkpatrick, Rev. Mod. Phys. 45, 574 (1973).

[3] B. Derrida and J. Vannimenus, J. Phys. A 15, L557-L564 (1982).

[4] A. B. Harris and T. C. Lubensky, Phys. Rev. B 35, 6964 (1987).

[5] A. Hansen and E.L. Hinrichsen, Phys. Rev. B 43, 665 (1991).

[6] Statistical Models for the Fracture of Disordered Media edited by H.J. Herrmann and S. Roux (Elsevier, Amsterdam,, 1990).

[7] L. de Arcangelis, S. Redner, and H.J. Herrmann, J. de Physique 46, L585 (1985). 
[8] P.M. Duxbury, P.D. Beale, and C. Moukarzel, Phys. Rev. B 51, 3476-3488 (1995).

[9] G.G. Batrouni, A. Hansen and G.H. Ristow, J. Phys. A 27, 1363 (1994).

[10] R. Albert and A.-L. Barabási, Rev. Mod. Phys. 74, 47 (2002).

[11] S.N. Dorogovtsev and J.F.F. Mendes, Adv. in Phys. 51, 1079 (2002).

[12] M.E.J. Newman, SIAM Review 45, 167 (2003).

[13] S.H. Strogatz, Nature 410, 268 (2001).

[14] M. Barahona and L.M. Pecora, Phys. Rev. Lett. 89, 054101 (2002).

[15] T. Nishikawa, A.E. Motter, Y.-C. Lai, and F.C. Hoppensteadt, Phys. Rev. Lett. 91, 014101 (2003).

[16] A.E. Motter, C. Zhou, and J. Kurths, Phys. Rev. E. 71, 016116 (2005).

[17] G. Grinstein and R. Linsker, Proc. Natl. Acad. Sci. USA 102, 9948 (2005).

[18] G. Korniss, M.A. Novotny, H. Guclu, and Z. Toroczkai, P.A. Rikvold, Science 299, 677 (2003).

[19] M. Argollo de Menezes and A.-L. Barabási, Phys. Rev. Lett. 92028701 (2004).

[20] Z. Toroczkai and K. Bassler, Nature 428, 716 (2004).

[21] A. Barrat, M. Barthelemy, R. Pastor-Satorras, and A. Vespignani, Proc. Natl. Acad. Sci. USA 101, 3747 (2004).

[22] K. Park, Y.-C. Lai, L. Zhao, and N. Ye, Phys. Rev. E 71, 065105(R) (2005).

[23] M.J. Berryman, A. Allison, and D. Abbott, in Noise in Communication, edited by L.B. White, Proceedings of SPIE Vol. 5473 (SPIE, Bellingham, WA, 2004) pp.122-130.

[24] D.J. Ashton, T.C. Jarrett, and N.F. Johnson, Phys. Rev. Lett. 94, 058701 (2005).

[25] R. E. Aitchison, Am. J. Phys. 32, 566 (1964).

[26] M.E.J. Newman, Phys. Rev. E 64, 016132 (2001).

[27] M.E.J. Newman and M. Girvan, Phys. Rev. E 69, 026113 (2004).

[28] F. Wu and B.A. Huberman, Eur. Phys. J. B. 38, 331 (2004).

[29] M.E.J. Newman, Social Networks 27, 39 (2005).

[30] E. López, S.V. Buldyrev, S. Havlin, and H.E. Stanley, Phys. Rev. Lett. 94, 248701 (2005).

[31] D.-S. Lee, H. Rieger, arXiv:cond-mat/0503008 (2005);

[32] A.-L. Barabási and R. Albert, Science 286, 509 (1999). 
[33] D.J. Watts and S.H. Strogatz, Nature 393, 440 (1998).

[34] D.J. Watts, Am. J. Soc. 105, 493 (1999).

[35] M.E.J. Newman, J. Stat. Phys. 101, 819 (2000).

[36] B. Kozma, M. B. Hastings, and G. Korniss, Phys. Rev. Lett. 92, 108701 (2004).

[37] B. Kozma, M. B. Hastings, and G. Korniss, Phys. Rev. Lett. 95, 018701 (2005).

[38] B. Kozma, M.B. Hastings, and G. Korniss, in Noise in Complex Systems and Stochastic Dynamics III, edited by L.B. Kish, K. Lindenberg, Z. Gingl, Proceedings of SPIE Vol. 5845 (SPIE, Bellingham, WA, 2005) pp.130-138.

[39] M. B. Hastings, Eur. Phys. J. B 42, 297 (2004).

[40] S.F. Edwards and D.R. Wilkinson, Proc. R. Soc. London, Ser A 381, 17 (1982).

[41] Z. Toroczkai, G. Korniss, M. A. Novotny, and H. Guclu, in Computational Complexity and Statistical Physics, edited by A. Percus, G. Istrate, and C. Moore, Santa Fe Institute Studies in the Sciences of Complexity Series (Oxford University Press, 2005, in press); arXiv:cond-mat/0304617.

[42] A. Nagurney, J. Cruz, J. Dong, and D. Zhang, Eur. J. Oper. Res. 26, 120 (2005).

[43] S. Kirkpatrick, Science 299, 668 (2003).

[44] F.Y. Wu, J. Phys. A 37, 6653 (2004).

[45] J. Cserti, Am. J. Phys. 68, 896 (2000).

[46] P.G. Doyle and J.L. Snell, Random Walks and Electric Networks, Carus Mathematical Monograph Series Vol. 22 (The Mathematical Association of America, Washington, DC, 1984), pp. 83-149; arXive: math.PR/0001057.

[47] L. Lovász, Random Walks on Graphs: A Survey in Combinatorics, Paul Erdős is Eighty Vol. 2, edited by D. Miklós, V.T. Sós, and T. Szőnyi (János Bolyai Mathematical Society, Budapest, 1996), pp. 353-398; http://research.microsoft.com/users/lovasz/erdos.ps.

[48] S. Redner, A Guide to First-Passage Processes (Cambridge University Press, Cambridge, UK, 2001).

[49] A.-L. Barabási and H.E. Stanley, Fractal Concepts in Surface Growth (Cambridge University Press, Cambridge, 1995).

[50] M. Anghel, Z. Toroczkai, K.E. Bassler, and G. Korniss, Phys. Rev. Lett. 92, 058701 (2004).

[51] P. Erdős and A. Rényi, Publ. Math. Inst. Hung. Acad. Sci. 5, 17 (1960).

[52] M.E.J. Newman and D.J. Watts, Phys. Lett. A 263, 341 (1999).

[53] R. Monasson, Eur. Phys. J. B 12, 555 (1999). 
[54] W.H. Press, S.A. Teukolsky, W.T. Vetterling, B.P. Flannery, Numerical Recipes in $C, 2$ nd ed. (Cambridge Univ. Press, Cambridge, 1995), Secs. 11.2 and 11.3.

[55] B. Kozma, M.B. Hastings, and G. Korniss, in preparation.

[56] For the BA scale-free model [32] (growth and preferential attachment), each new node is connected to the network with $m$ links, resulting in an average degree of $2 m$ in the large- $N$ limit.

[57] M. B. Hastings, Phys. Rev. Lett. 91, 098701 (2003).

[58] S. Jespersen and A. Blumen, Phys. Rev. E 62, 6270 (2000).

[59] R. T. Scalettar, Physica A 170, 282 (1991).

[60] A. Barrat and M. Weigt, Eur. Phys. J. B 13, 547 (2000).

[61] M. Gitterman, J. Phys. A 33, 8373 (2000).

[62] B.J. Kim, H. Hong, P. Holme, G.S. Jeon, P. Minnhagen, and M.Y. Choi, Phys. Rev. E 64, 056135 (2001).

[63] H. Hong, B.J. Kim, and M.Y. Choi, Phys. Rev. E 66, 018101 (2002).

[64] C. P. Herrero, Phys. Rev. E 65, (2002) 066110.

[65] M.A. Novotny and S.M. Wheeler, Braz. J. Phys. 34395 (2004);

[66] D. Jeong, H. Hong, B.J. Kim, and M.Y. Choi, Phys. Rev. E 68, 027101 (2003).

[67] R. Albert. H. Jeong, and A.-L. Barabási, Nature 406, 378 (2000).

[68] R. Cohen, K. Erez, D. ben-Avraham, S. Havlin, Phys. Rev. Lett. 85, 4626 (2000).

[69] R. Cohen, K. Erez, D. ben-Avraham, S. Havlin, Phys. Rev. Lett. 86, 3682 (2001). 